\title{
Wireless Link Simulations using Multi-level Markov Models
}

Ankur U. Kamthe, Miguel Á. Carreira-Perpiñán and Alberto E. Cerpa University of California, Merced

\section{Introduction}

Modeling the behavior of 802.15 .4 links is a non-trivial problem because of the widespread heterogeneity in the quality of any given link over time. Moreover, links experience different leve of dynamics at short and long time scales, which makes the design of a suitablimodel that combines the different dynamics at different timescales bines the different dynamics at different timescales a difficult task.

\section{Contribution}

We introduce novel multilevel approach involving Hidden Markov Models (HMMs)[1] and Mixtures of Multivariate Bernoullis (MMBs)[2] for modeling the long and short time scale behavior of links in wireless sensor networks.

\section{Modeling Approach

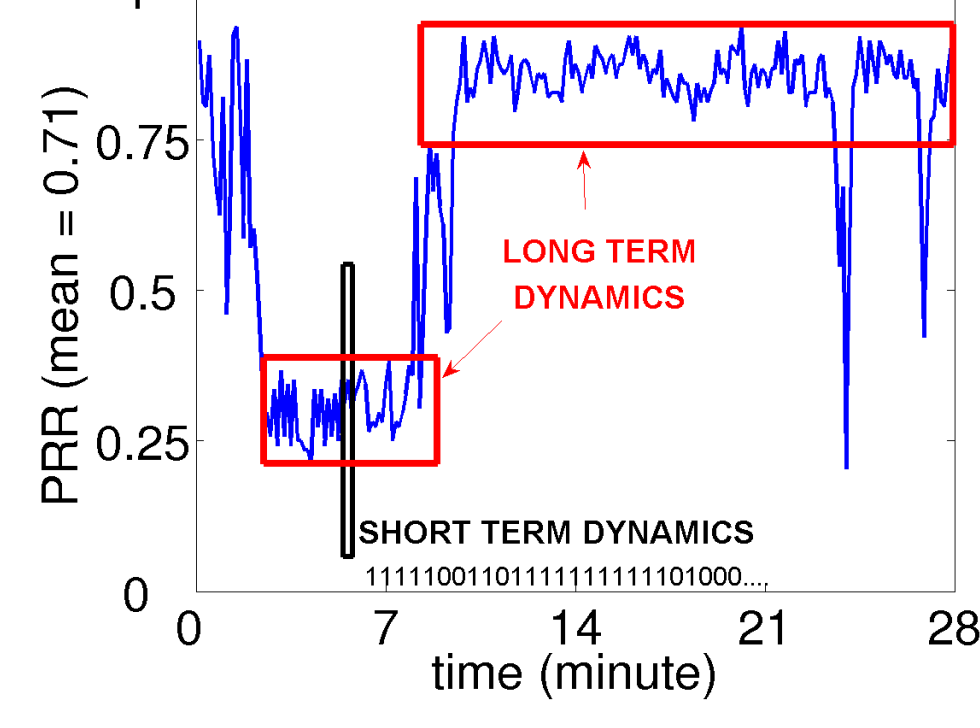

(a) Vacket Reception of a link over time

The fundamental motivation for our modeling approach is that observed traces display structure at different temporal scales.

- Long-term dynamics: From Figure (a), we observe that over a period of minutes the link seems to switch between two states: one with $\mathrm{PRR} \approx 0.3$ and the other with $\mathrm{PRR} \approx 0.86$.

- Short-term dynamics are variations in consecutive packet reception successes or failures. In a period of seconds, however, while the PRR may stay roughly constant at 0.3 , it is more likely to observe a bursty sequence 000000001111 than a wildly oscillating sequence 010010100100 .

- For realistic simulation, the model must replicate the multiscale structure.

\section{Multi-level Markov (M\&M) Model}

$00011111 \frac{10001110}{A} \frac{00011111}{A} \frac{00011110}{A}$

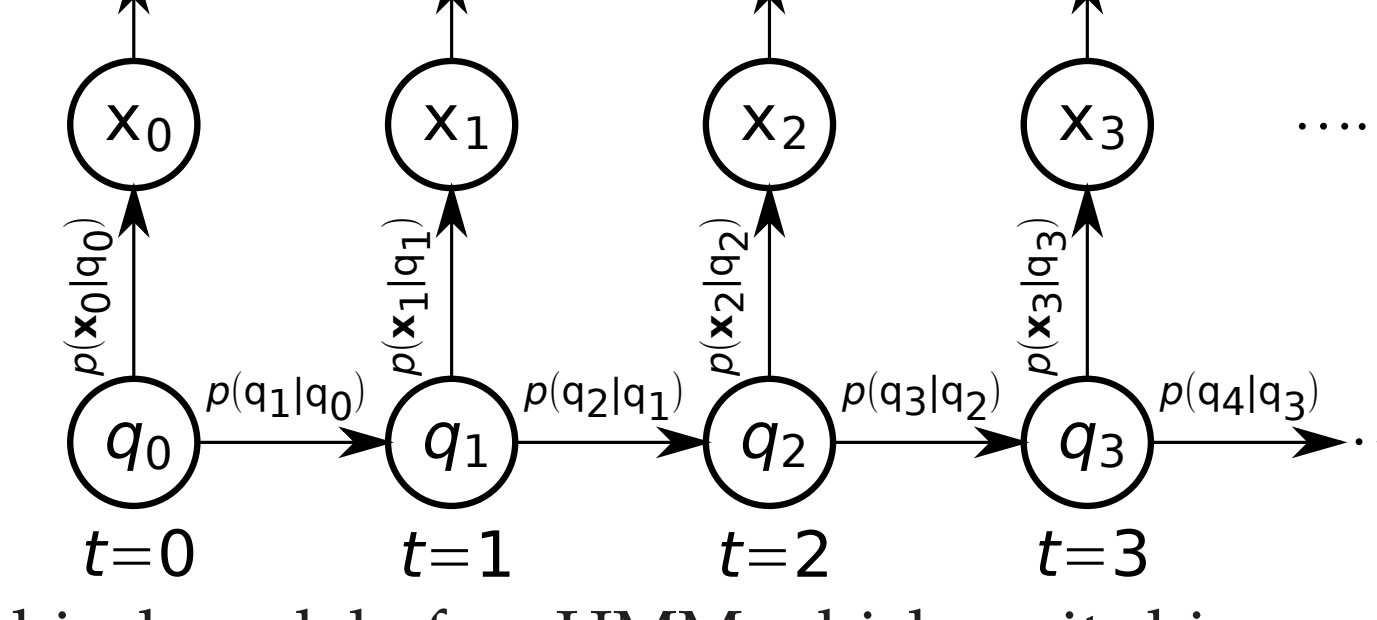

(b) Graphical model of an HMM which emits binary strings $\mathrm{x}_{t}$ length 8 . In the M\&M model, this is the L1-HMM, and $p\left(\mathbf{x}_{t} \mid q_{t}\right)$ is itself an HMM or an MMB

- The data trace is modeled as a sequence of binary strings (windows) $\mathbf{x}_{t}$ of length $W$ (see Figure (b)). $W$ controls the tradeoff of short vs. long term.

- Level-1 hidden Markov model (L1-HMM) with $q=1, \ldots, Q_{1}$ states models transitions between long-term dynamics. Each long-term state $q$ has its own distribution $p(\mathbf{x} \mid q)$ of emitting binary $W$

1. A hidden Markov model (L2-HMM). This has sociated transition probability matrix, and (2) a (univariate) Bernoulli emission distribution with parameter $p$

2. A mixture of multivariate Bernoulli distributions $(L 2-M M B)$. This mixture has $M$ components, and each component has $W+1$ parameters: a mixture proportion and a vector $\mathbf{p}=\left(p_{1}, \ldots, p_{W}\right)^{T}$ of Bernoulli parameters.

- Learning: Estimating the parameters of the $\mathrm{M} \& \mathrm{M}$ model is done by maximizing the loglikelihood of the given data set over all the model parameters. windows, capturing the short-term behavior.

\section{- Level-2 or short-term models:} (1) a set of $Q_{2}$ short-term states and its as-

\section{Model Evaluation}

- For each link, learn model parameters given data traces of length 230,400 $\left(Q_{1}=6, Q_{2}=2\right.$ and $\left.M=20\right)$.

- For each model, sample a long sequence and compared performance on the basis of: (1) Packet Reception Rates (PRR), (2) Distributions of run lengths of 1's and 0's, and (3) Conditional Packet Delivery Function (CPDF).

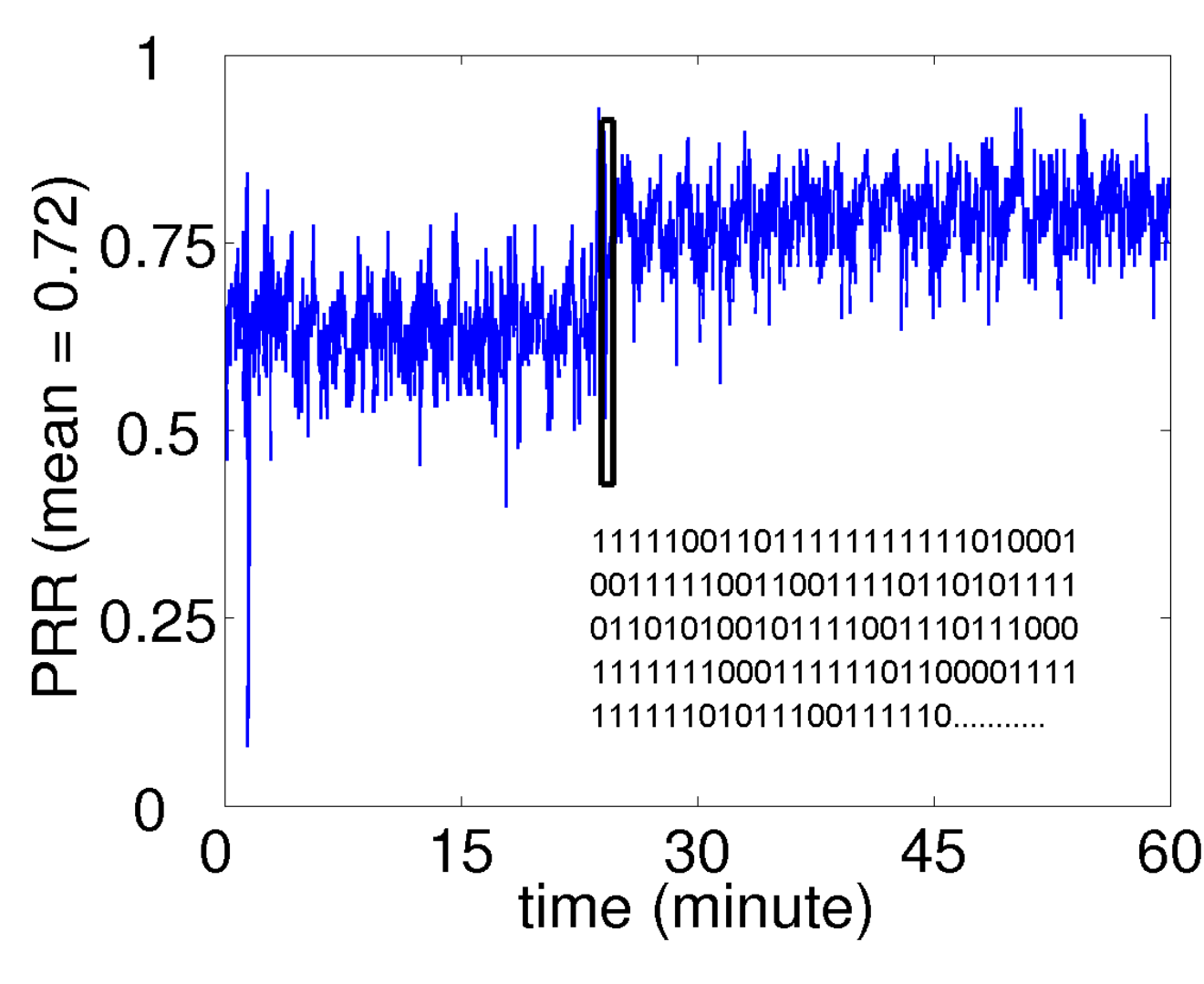

(c) PRR variation of original trace

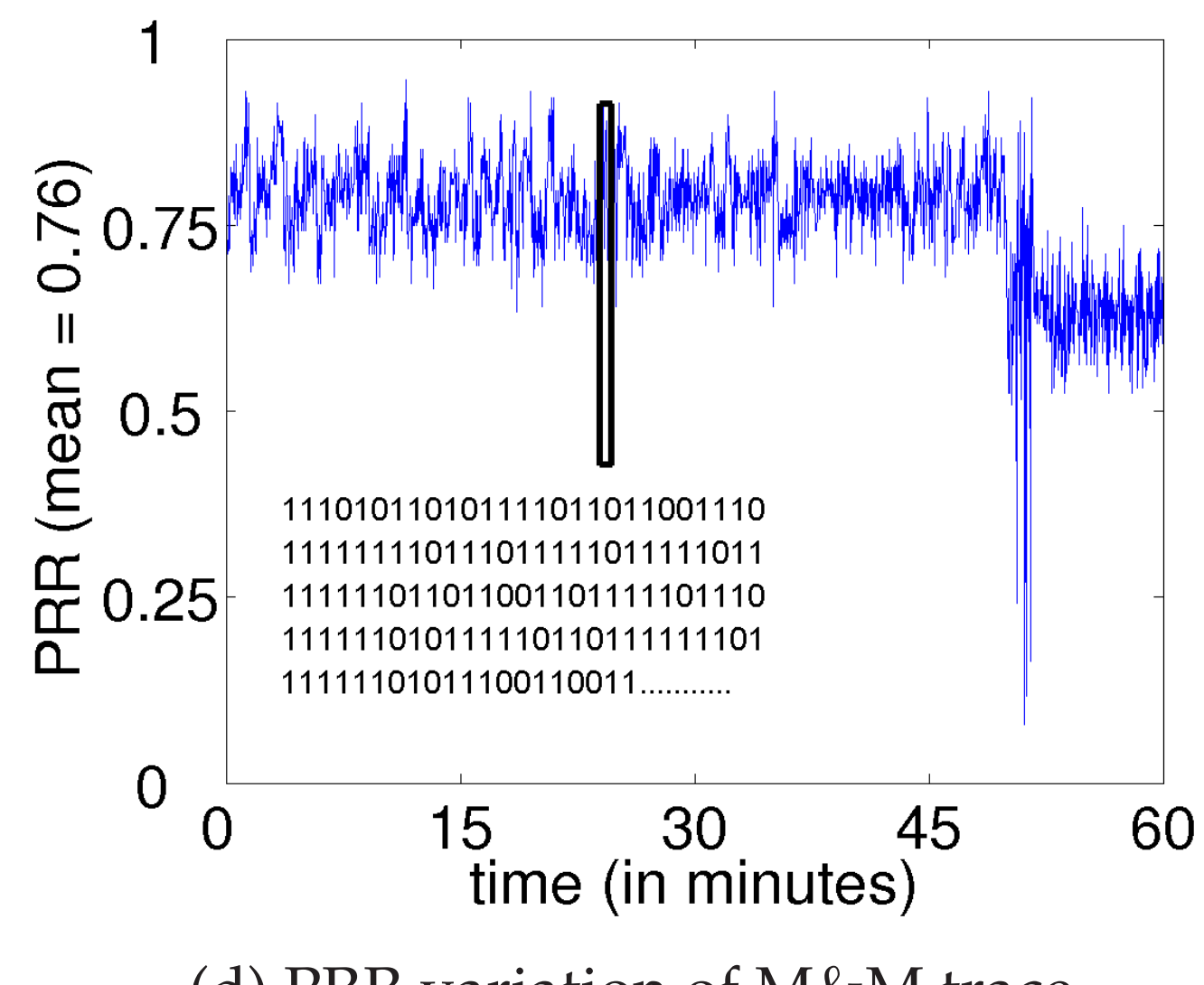

(d) PRR variation of M\&M trace
- The simulated traces (see Figure (d)) are able to capture the long term dynamics quite accurately when compared to the original traces ( see Figure (c)

- Overall, the average difference between the PRR of the simulated and the original trace was less than $2.5 \%$ whereas the average standard deviation of the simulated link PRR was 0.004
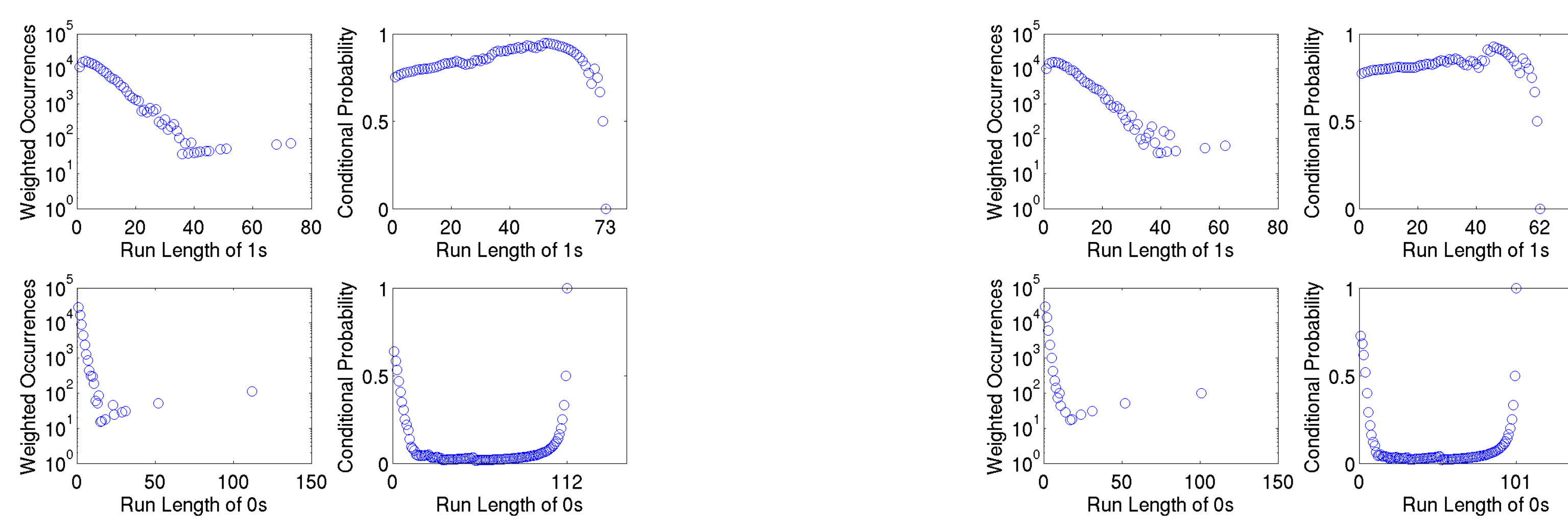

(e) Distribution of Run lengths and CPDFs for original trace (f) Distribution of Run lengths and CPDFs for M\&M trace

- Figure (e) and (f) plot the distributions of run lengths and CPDFs of 0's and 1's for the original and sim- We can see that the M\&M traces are able to simulate the longer runs/bursts as seen in the training trace.

\section{Summary and Future Work}

- Our model allows us to learn from data, not just bursts but far more complex behaviors. For more details, refer to [3]

- The M\&M model is a generalization of the Gilbert model [4]

Transforming existing model parameters to simulate new environments using order of magnitude less training samples by applying model adaptation techniques [5] is part of our future research agenda.

- The model can be extended to emit signal strength values, thus, modeling physical layer characteristics such as RSSI values of wireless traces.

\section{References}

[1] C. Bishop Pattern Recognition \& Machine Learning, 2006 M. A. Carreira-Perpiñán, S. Renals Practical Identifiability Neural Computation, 2000 Multivariate Bernoulli Distribution A. Kamthe, M. Á. Carreira-Perpiñán, A. Cerpa M\&M:

4] E. N. Gilbert Capacity of a burst-
tem Technical Journal, Vol. 39,1960 P. C. Woodland Speaker adaptation for continuous den
HMMs: A review Invited Lecture, In Adaptation, 2001 Multi-level Markov Model for Wireless Link Simulations In 